\title{
Survival analysis to assess of the effectiveness of fecal microbiota transplantation for recurrent and/or refractory Clostridioides difficile infection
}

\author{
Tatiana Petukhova ${ }^{1 *}$, Peter T. Kim ${ }^{1,2}$ and Anthony F. Desmond ${ }^{1}$
}

*Correspondence: tpetukho@uoguelph.ca

\author{
CrossMark
}

$\leftarrow$ Click for updates

'Department of Mathematics and Statistics, University of Guelph, Guelph, Canada.

2Department of Pathology and Molecular Medicine, McMaster University, Hamilton, Canada.

\begin{abstract}
Background: Clostridioides difficile infection causes chronic and sometimes life-threatening diarrhea in patients as a consequence of antibiotics overuse. A promising experimental procedure for recurrent and/ or refractory $C$. difficile infection is fecal microbiota transplantation therapy. The aim of this study was to analyze medical records of patients infected with recurrent and/or refractory $C$. difficile that were treated with fecal microbiota transplantation therapy to investigate the relationship between time to clinical resolution and explanatory variables.

Methods: The analyses were based on a retrospective review of patients' data. Data of ninety-two patients between 24 and 95 years of age of which $43.6 \%$ were males were available for analyses. Three variables, age group, gender and hospitalization status, were included in the analyses. For time-to-event endpoints, the comparison between two groups was done with the Kaplan-Meier estimator. The nonparametric logrank test was used to compare the survival distributions between two age groups. The Cox proportional hazard model was used to analyze age, gender and hospitalization status as risk factors to clinical resolution. The most satisfactory model was selected based on the value of Akaike's information criterion. The proportional hazard assumptions and the overall model fit were assessed based on graphical evidence, hypothesis testing and residual analyses.

Results: Overall, clinical resolution was achieved for $92 \%$ of the patients. In fact, $95.7 \%$ of them in the age group younger than 65 years and $83.1 \%$ in the age group 65 years and older achieved clinical resolution. We found that the hazard of fecal microbiota transplantation to $C$. difficile in patients younger than 65 is twice as high as in patients who were 65 and older. On average, the age group younger than 65 years received 1.3 fecal microbiota transplantations, while the older age group received 2.2 fecal microbiota transplantations. Results of analyses indicate that the used models were appropriate.

Conclusion: Delivery of fecal microbiota transplantation via a retention enema is an effective alternative therapy for recurrent and/or refractory $C$. difficile infection. Age is strongly associated with clinical resolution, with older patients requiring more fecal microbiota transplantations and more time to be clinically resolved. The Kaplan-Meier estimators and the Cox proportional hazard model are adequate models to analyze data of patients infected with recurrent and/or refractory C. difficile. Randomized control trials with more variables are needed to confirm our findings and more deeply investigate the impact of other risk factors on clinical resolution.
\end{abstract}

Keywords: Clostridioides difficile infection, fecal microbiota transplantation, log-rank test, Kaplan-Meier estimator, Cox proportional hazard model, residual analysis

(c) 2020 Petukhova et al; licensee Herbert Publications Ltd. This is an Open Access article distributed under the terms of Creative Commons Attribution License (http://creativecommons.org/licenses/by/3.0). This permits unrestricted use, distribution, and reproduction in any medium, provided the original work is properly cited. 
Petukhova et al., Journal of Medical Statistics and Informatics 2020,

\section{Introduction}

Clostridioides difficile (C. difficile) infection (CDI) commonly occurs as a complication of antibiotic therapy. The healthy bacteria that reside within the colon are the major defense against the growth of $C$. difficile within the large intestine. Use of antibacterial medications facilitates the emergence of bacterial populations, causing the disruption of normal colonic flora. C. difficile takes this opportunity to multiply, produce toxins and cause $C$. difficile associated diarrhea (CDAD). Studies have shown that the overuse of almost all antibacterial medications is associated with CDAD [1]. Unlimited use of antibiotics leads to increased resistance to this conventional therapy [2] and causes the persistent disruption of healthy colonic flora, resulting in treatment failures with standard therapy and recurrence of disease. The economic impact associated with CDI is considerable with respect to direct health cost and loss of productivity of infected individuals. A promising experimental procedure for recurrent and/or refractory CDI involves infusion of intestinal microorganisms from healthy donors directly into the colon of infected subjects. This procedure is known as fecal microbiota transplantation (FMT) therapy. The aim of this study was to analyze medical records of patients affected with recurrent and/or refractory CDI and treated with FMTs to investigate the relationship between time to clinical resolution and explanatory variables. Survival analysis techniques were used to quantitatively describe the main features of collected records and to maximize insight into the dataset while uncovering underlying structure. We extracted relevant variables and detected outliers and anomalies. A parsimonious model with optimized factor settings was developed and underlying assumptions were verified. The information generated from these analyses was compared with reported research findings from other studies, where FMT was used to treat patients infected with $C$. difficile.

\section{Materials and Methods Ethics Statement}

The institutional ethics review boards approved the study of patients' records. All participants provided the approved informed consent.

\section{Data}

This is a retrospective study of patients who were recruited for the management of recurrent and/or refractory CDI. All patients met the Infectious Diseases Society of America (IDSA) criteria for CDI [3]. Data were collected from medical records from 94 adults aged between 24 and 95 years who underwent FMT via a retention enema between 2008 and 2012 at St.Joseph's Healthcare, Hamilton, Canada [4]. Some individuals did not respond to the first administered treatment and were given subsequent FMTs with antibiotics in between FMTs until clinical resolution. Clinical resolution for the purposes of this study means that participants cleared their diarrhea symptoms even though they may have still tested positive for $C$. difficile.

\section{Statistical Methods for Survival Data}

Descriptive statistics, exploratory data analysis techniques and confirmatory data analysis techniques were used to discover new features in the CDI patients' data. Subjects were classified into two age groups ( $<65$ and $\geq 65$ ) to verify reported claims from different studies about the effect of age on cure rates. For each category of patient variables, descriptive baseline statistics consist of sample size (n), percentage, mean, standard deviation, median and range. Summary tables are presented by age group and baseline characteristics. The two groups were compared by the chi-square or Fisher's exact tests for proportions.

For time-to-event endpoints, the Kaplan-Meier (K-M) estimator was used to compare the two groups. A Cox proportional hazard $(\mathrm{PH})$ model was used to investigate the effect of explanatory variables on time to clinical CDI resolution. The nonparametric log-rank test was used to compare the survival distributions between the two age groups. Estimation and inference were made without assuming any distribution for CDI survival time.

\section{Kaplan-Meier Estimator of the Survival Functions}

The K-M estimator, $\hat{S}(t)$, is a nonparametric method for estimating a survival function, $S(t)$, from lifetime data. The product of estimated survival probabilities resulting from Equation 1 forms the estimator

$$
\hat{S}(t)=\prod_{t_{i}<t}^{k} \frac{n_{i}-d_{i}}{n_{i}}
$$

where $t_{1}<t_{2}<\ldots<t_{k}$ are the rank-ordered recorded times among independent subjects, denoted as $\left(t_{i}, c_{i}\right), i=1,2, \ldots, n$ , given that the ith subject is able to survive prior to time $t_{i}$ , but at time $t_{i}$ this subject experiences an event of interest and $c_{i}$ is a censoring indicator variable with value of 1 for the observed event for the ith subject; otherwise, $0 ; n_{i}$ is the number of subjects at risk just prior to $t_{i} ; d_{i}$ is the number of subjects that experience the event at $t_{i}$.

\section{The Log-Rank Test}

The Log-Rank test is a nonparametric test for a difference of survival functions, using the G-rho family of tests [5]. The test is used when the hazard functions are thought to be proportional across the groups. The test statistic is constructed by giving equal weights rho to the contribution of each event time of $S(t)^{r h o}$ to the overall test statistic, where $S(t)$ is the K-M estimate of survival.

\section{Cox Proportional Hazards Regression Model}

A Cox PH regression model is used to quantify the effects of explanatory variables on the hazard of C. difficile. This particular form of regression model was proposed by Cox [6]. Under his model, the hazard function, $\lambda_{i}$, for the $i$ th subject is expressed as the product of two functions as given in 
Equation 2

$$
\lambda_{i}\left(t ; \beta, \mathrm{X}_{i}\right)=\lambda_{0}(t) \exp \left(X_{i}^{\prime} \beta\right)
$$

where $\lambda_{0}(\cdot)$ is an arbitrary unspecified baseline hazard function that characterizes how the hazard function changes as a function of time; $\exp \left(X_{i}^{\prime} \beta\right)$ is a function that characterizes how the hazard function changes as a function of regressor variables; $\beta=\{\beta\}^{p p}{ }_{j=1}$ is a $p \times 1$ vector of regression parameters; $X_{i}=\left\{X_{j}\right\}_{j=1}^{p}$ is a $p$-dimensional completely observed variable vector for the ith individual; a prime ( ) indicates transposition. Under the Cox PH model, time $t$ does not have any distributional constraints and the model is referred to as the $\mathrm{PH}$ model because the hazard functions are multiplicatively related. That is, their ratios are constant over time. Parameters under the Cox model are estimated from the partial likelihood function that is defined in Equation 3

$$
L(\beta)=\prod_{i: c_{i}=1} \frac{\exp \left(x_{i}^{\prime} \beta\right)}{\sum_{j \in R\left(t_{i}\right)} \exp \left(x_{j}^{\prime} \beta\right)}
$$

where $c_{i}$ is a censoring indicator variable with value of 1 for an observed event for the ith subject; otherwise, $0 ; R\left(t_{i}\right)$ is the risk set of all individuals with event times greater than or equal to time $t_{i}$.

To define the ratio of the hazard functions, let $X_{1}$ be a dichotomous variable with a value of either $X_{1}=1$ for group 1 or $X_{1}=0$ for group 2, where group 2 is a reference category. Then under the model 2, the ratio of the hazard functions for the two groups, after adjusting for other variables, is Equation 4

$$
\operatorname{HR}(t)=\frac{\lambda_{\text {group } 1}(t ; \beta, X)}{\lambda_{\text {group } 2}(t ; \beta, X)}=\frac{\lambda_{0}(t) \exp \left(\beta_{1}+\beta_{2} x_{2}+\ldots+\beta_{p} x_{p}\right)}{\lambda_{0}(t) \exp \left(\beta_{2} x_{2}+\ldots+\beta_{p} x_{p}\right)}=\exp \left(\beta_{1}\right)
$$

The hazard ratio depends only on the function $\exp \left(\beta_{1}\right)$ and the actual form of the baseline hazard function is of little importance.

\section{Model Development}

In this study, the failure time of recurrent and/or refractory CDI in days, i.e. time to clinical resolution of individuals, was considered as the response variable. Time for the first successful FMT was taken to be one day. For subjects treated with more than one FMT, time was counted from the date of the first administered FMT to clinical resolution. For subjects who did not achieve clinical resolution or who were lost to follow-up prior to CDI resolution, time was right censored at the date of the last given FMT. To identify if a case was censored, an indicator variable was assigned as 1 for subjects whose clinical resolution was not observed and 0 , otherwise. Along with this, the following three completely recorded classification variables were taken into consideration:

1. Age group (younger than 65 years versus 65 years and older)

2. Gender (female versus male)
3. Hospitalization (inpatient versus outpatient at the date of FMT)

\section{Model Assessment}

Model adequacy was examined to evaluate how well the fitted regression surface characterized the data. The most satisfactory model was selected based on the forward selection procedure and the smallest value of Akaike's information criterion (AIC). The PH assumptions and the overall model fit were investigated based on graphical evidence and hypothesis tests. The parallel nature of the Kaplan-Meier estimated curves for a given variable in a log-log plot was assessed. Scaled Schoenfeld residuals were used to test for the constancy of the coefficients.

The martingale residuals were used from the Cox model to detect possible outliers. The values of the martingale residuals are the differences between the observed events (observed clinical resolution) and the predicted events, conditional on the fitted model, follow-up time and the observed variable. Negative martingale residuals either represent censored observations or indicate that the observed number is less than expected. Positive martingale residuals suggest than the observed number is bigger than expected.

The deviance residuals were used to validate the model accuracy and possible outliers. These residuals are a normalizing transform of the martingale residuals and are symmetric about zero when a fitted model is appropriate.

The score residuals were used to assess influence of each point on the estimated $\beta$ coefficients. The change in the estimated coefficients of $\beta$ was computed by dropping an observation. The obtained value was then divided by the standard errors for the components of the estimated coefficient.

All analyses were performed using the R statistical software, version 3.5.3 [7]. For all statistical analyses, $p<0.05$ was considered statistically significant.

\section{Results \\ Descriptive Statistics}

The analysis was based on a retrospective review of 41 (43.6\%) male and 53 (56.4\%) female patients. At the time of FMT, the majority of patients ( $n=70,74.5 \%$ ) were hospitalized. The mean (standard deviation) age of the patients was 71.8 (15.7) years, ranging from 24 to 95 years. Clinical resolution was achieved for $86(92 \%)$ subjects. There was no significant difference in the cure rates between males and females $\left(\chi^{2}=1.22 ; \mathrm{df}=1\right.$; $p=0.3)$ and between outpatients and inpatients $\left(\chi^{2}=0.05 ; \mathrm{df}=1\right.$; $p=0.8$. The Fisher's exact test indicated that the clinical resolution rate in the group with older patients was not significantly different from the clinical resolution rate in the group with younger patients $(p=0.2)$. The proportion of patients with recurrent and/or refractory CDI in the age groups was significantly different $\left(\chi^{2}=47 ; \mathrm{df}=1 ; p<0.01\right)$. On average, the age group younger than 65 years received 1.3 FMTs, while the age group 65 years and older was treated with 2.2 FMTs $(t=-4.3 ; p<0.01 ; 95 \% \mathrm{Cl},-1.3$ to -0.5$)$. 
Baseline characteristics for each age group are summarized in Tables 1 and 2 . In the age group younger than 65 years, 22 patients out of 23 (95.7\%) were clinically resolved, 16 with 1 FMT, 5 with 2 FMTs, and 1 with 3 FMTs. There was no significant predominance of females $(n=13 ; 56.5 \%)$ or inpatients $(n=12$; $52.2 \%)$. The mean age (standard deviation) of the patients in this group was 49 (12.2) years with an age range of 24-63 years. In the age group 65 years and older, 59 patients out of 71 (83.1\%) achieved clinical resolution of CDI. In this group, 29 patients were successfully resolved with $1 \mathrm{FMT}, 13$ patients with 2 FMTs, 12 patients with 3 FMTs, 3 patients with 4 FMTs and 2 patients with 6 FMTs. There was no significant female predominance $(n=40 ; 56.3 \%)$ and the majority of patients $(n=58 ; 81.7 \%)$ were hospitalized. The mean age (standard deviation) of the patients was 79.1 (7.3) years with an age range of 65-95 years.

\section{The Cox PH Regression Model}

A Cox PH regression model was fitted to the data along with three classification variables. The variable selection procedure selected Age group as a significant predictor. Using Equation 2 , the hazard function for the $i$ th patient at any given time $t$ can be written as

$$
\lambda_{i}(\mathrm{t})=\lambda_{0}(\mathrm{t}) \exp (\beta \text { Age.group } 2)
$$

where Age.group $2=1$ for the patients aged 65 years and older; Age.group $2=0$ for the patients aged younger than 65 years. The estimate of $\beta$ and its standard error were obtained by using the Efron approximation as this approximation provides an improved solution in the presence of ties. The computation results are summarized in Table 3 . There was a significant difference in clinical resolution between older and younger participants ( $H R=0.53 ; 95 \% \mathrm{Cl}, 0.32$ to $0.88 ; p=0.01$ by the Wald test).

Table 1. Baseline characteristics for the patients aged younger than $\mathbf{6 5}$ years infected with recurrent and/or refractory Clostridioides difficile infection and treated with fecal microbiota transplantation.

\begin{tabular}{|c|c|c|c|c|c|c|}
\hline \multirow[t]{2}{*}{ Characteristic } & \multirow{2}{*}{$\begin{array}{l}\text { Sample Size, } \\
\text { n (\%) }\end{array}$} & \multirow{2}{*}{$\begin{array}{l}\text { Clinical Resolution } \\
(\%)\end{array}$} & \multicolumn{4}{|c|}{ Age } \\
\hline & & & Mean & $\begin{array}{l}\text { Standard } \\
\text { Deviation }\end{array}$ & Median & Range \\
\hline \multicolumn{7}{|l|}{ Gender: } \\
\hline Males & $10(43.5 \%)$ & $10(100 \%)$ & 54.4 & 5.4 & 55 & $47-63$ \\
\hline Females & $13(56.5 \%)$ & $12(92.3 \%)$ & 44.9 & 14.4 & 49 & $24-63$ \\
\hline \multicolumn{7}{|l|}{$\begin{array}{l}\text { Hospitalization } \\
\text { Status: }\end{array}$} \\
\hline Inpatients & $12(52.2 \%)$ & $12(100 \%)$ & 55 & 7.1 & 56 & $40-63$ \\
\hline Outpatients & $11(47.8 \%)$ & $10(90.9 \%)$ & 42.5 & 13.5 & 48 & $24-61$ \\
\hline Total & $23(24.5 \%)$ & $22(95.7 \%)$ & 49 & 12.2 & 54 & $24-63$ \\
\hline
\end{tabular}

Table 2. Baseline characteristics for the patients aged 65 years and older infected with recurrent and/or refractory Clostridioides difficile infection and treated with fecal microbiota transplantation.

\begin{tabular}{|c|c|c|c|c|c|c|}
\hline \multirow[t]{2}{*}{ Characteristic } & \multirow{2}{*}{$\begin{array}{l}\text { Sample Size, } \\
\text { n (\%) }\end{array}$} & \multirow{2}{*}{$\begin{array}{l}\text { Clinical Resolution } \\
\text { (\%) }\end{array}$} & \multicolumn{4}{|c|}{ Age } \\
\hline & & & Mean & $\begin{array}{l}\text { Standard } \\
\text { Deviation }\end{array}$ & Median & Range \\
\hline \multicolumn{7}{|l|}{ Gender: } \\
\hline Males & $31(43.7 \%)$ & $23(74.2 \%)$ & 80.1 & 7.6 & 80 & $66-95$ \\
\hline Females & $40(56.3 \%)$ & $36(90 \%)$ & 78.4 & 7.1 & 80 & $65-90$ \\
\hline \multicolumn{7}{|l|}{$\begin{array}{l}\text { Hospitalization } \\
\text { Status: }\end{array}$} \\
\hline Inpatients & $58(81.7 \%)$ & $48(82.8 \%)$ & 80.2 & 7.1 & 81.5 & $65-95$ \\
\hline Outpatients & $13(18.3 \%)$ & $11(84.6 \%)$ & 74.5 & 6.6 & 76 & $66-90$ \\
\hline Total & $71(75.5 \%)$ & $59(83.1 \%)$ & 79.1 & 7.3 & 80 & $65-95$ \\
\hline
\end{tabular}

Table 3. Estimated coefficient for the Cox PH model to determine the effect of age group classification variable on clinical resolution of recurrent and/or refractory Clostridioides difficile infection.

\begin{tabular}{|c|c|c|c|c|c|c|c|}
\hline \multirow{2}{*}{ Variable } & \multirow[t]{2}{*}{$\hat{\beta}$} & \multirow{2}{*}{$\operatorname{SE}(\hat{\beta})$} & \multirow[t]{2}{*}{$\operatorname{Exp}(\hat{\beta})$} & \multirow[t]{2}{*}{$\mathrm{Z}$} & \multirow{2}{*}{ p-value } & \multicolumn{2}{|c|}{$95 \%$ CI for $\beta$} \\
\hline & & & & & & Lower & Upper \\
\hline Age.group 2 & -0.64 & 0.26 & 0.53 & -2.48 & 0.01 & 0.32 & 0.88 \\
\hline
\end{tabular}


The hazard of FMT to recurrent and/or refractory C. difficile was half as low in patients who were 65 years and older compared to patients who were younger than 65 years.

\section{PH Assumptions}

It was assumed that the hazard of the disease at any given time for an individual in one group was proportional to the hazard at that time for a similar individual in the other group. To verify this assumption, graphical and formal assessments were performed. Figure 1 shows the Kaplan-Meier estimated survival curves on the log-log scale, which is the hazard function for recurrent and/or refractory C. difficile on the log scale, in patients aged younger than 65 years and in patients aged 65 years and older. This plot provides evidence that the proportional hazards assumption between the two age groups is valid. In particular, the hazard to recurrent and/or refractory C. difficile is significantly greater in younger patients than in older patients $(p=0.02)$.

The results for the constancy of the coefficient for Age. group indicate that there is significant evidence that the coefficient is not significantly different from 0 and does not vary with time $(p=0.76)$.

\section{Model Fit}

A complete and thorough examination of model adequacy was performed. The diagnostic procedures were based on the analysis of residuals. The martingale and deviance residuals were calculated to verify the model accuracy and possible outliers. A scatterplot of the martingale residuals is shown in Figure 2A, where dots represent individual subjects. One noticeable feature of the plot is that there is a large number of residuals with a value of 0.7 due to the large number of ties (45 patients achieved clinical resolution within one day). Observations 30, 38 and 40 have the largest residuals.

The plot of the deviance residuals against the observation numbers in Figure 2B shows a similar feature as reported in Figure $2 \mathrm{~A}$. There is a horizontal band of points above zero because of tied events. Observations 30, 38 and 40 have the largest residuals.

The score residuals in Figure $2 \mathrm{C}$ are plotted to assess individual influences on the estimated coefficients. The plot shows that most estimates of the parameters are changed by less than 0.2 standard errors. Observations 38,41 and 80 with a value of more than 0.38 standard errors are the possible influential observations on the estimated coefficient.

\section{Discussion}

Antibiotic treatments often result in high rate of $C D I$ recurrence in patients. Between 15 and $30 \%$ of individuals affected by CDI experience recurrence after initial successful treatment with antibacterial treatments [8-10]. Overuse of antibiotics also leads to the development of resistance and treatment failure with standard therapy. Fecal microbiota transplantation (FMT) has emerged as a second-line therapy to treat recurrent and refractory CDI. In our study, we found, as reported in Lee et al. [4], that most patients were CDI resolved (92\%). This high success rate falls within previously published studies reporting cure rates ranging from 50 to $100 \%$ for FMT treated patients $[11,12]$. The wide range in success rate in studies reported in the literature may be explained, at least partially, by differences in sample size. Total success rate (100\%) was achieved in studies with a small sample size (1 to 7 patients) $[11,12]$. Ours is one of the studies with larger sample sizes of CDI patients treated with FMT so far reported, and $92 \%$ clinical resolution rate sup-

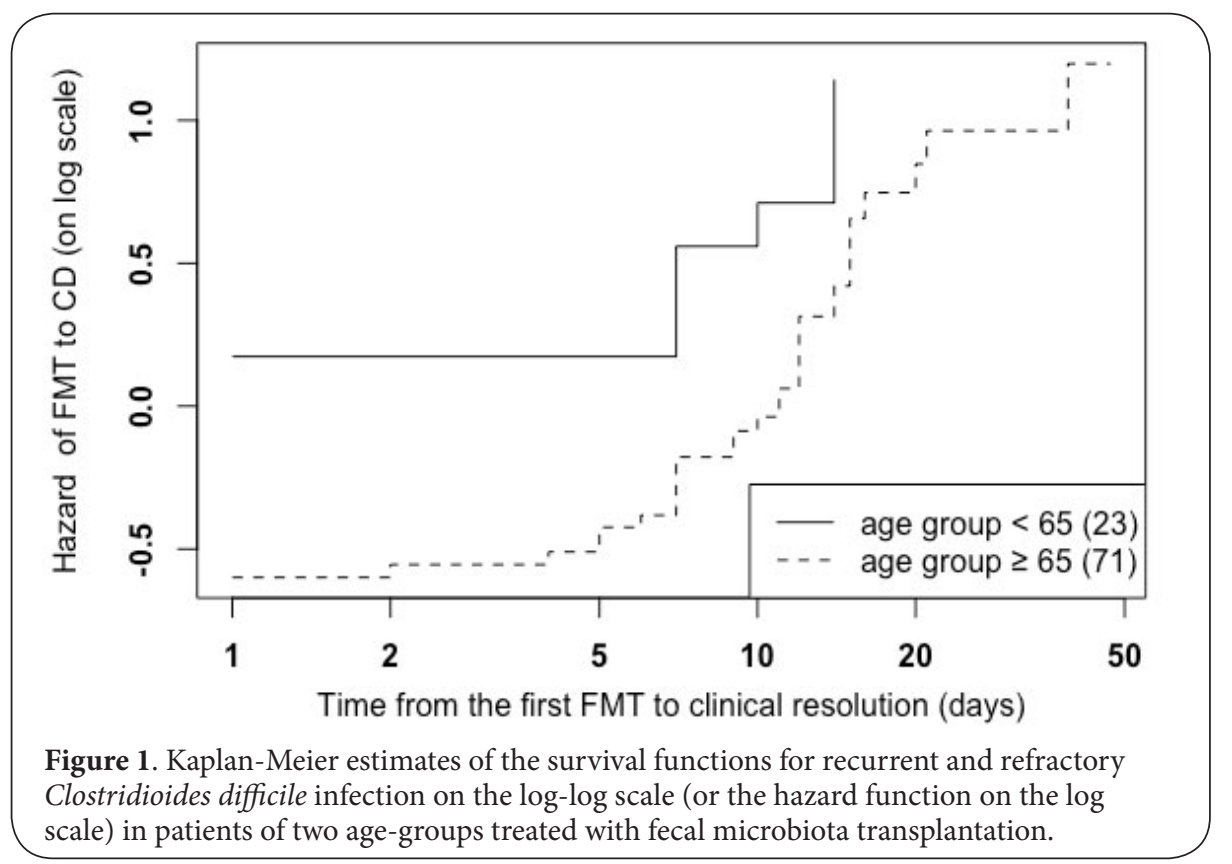




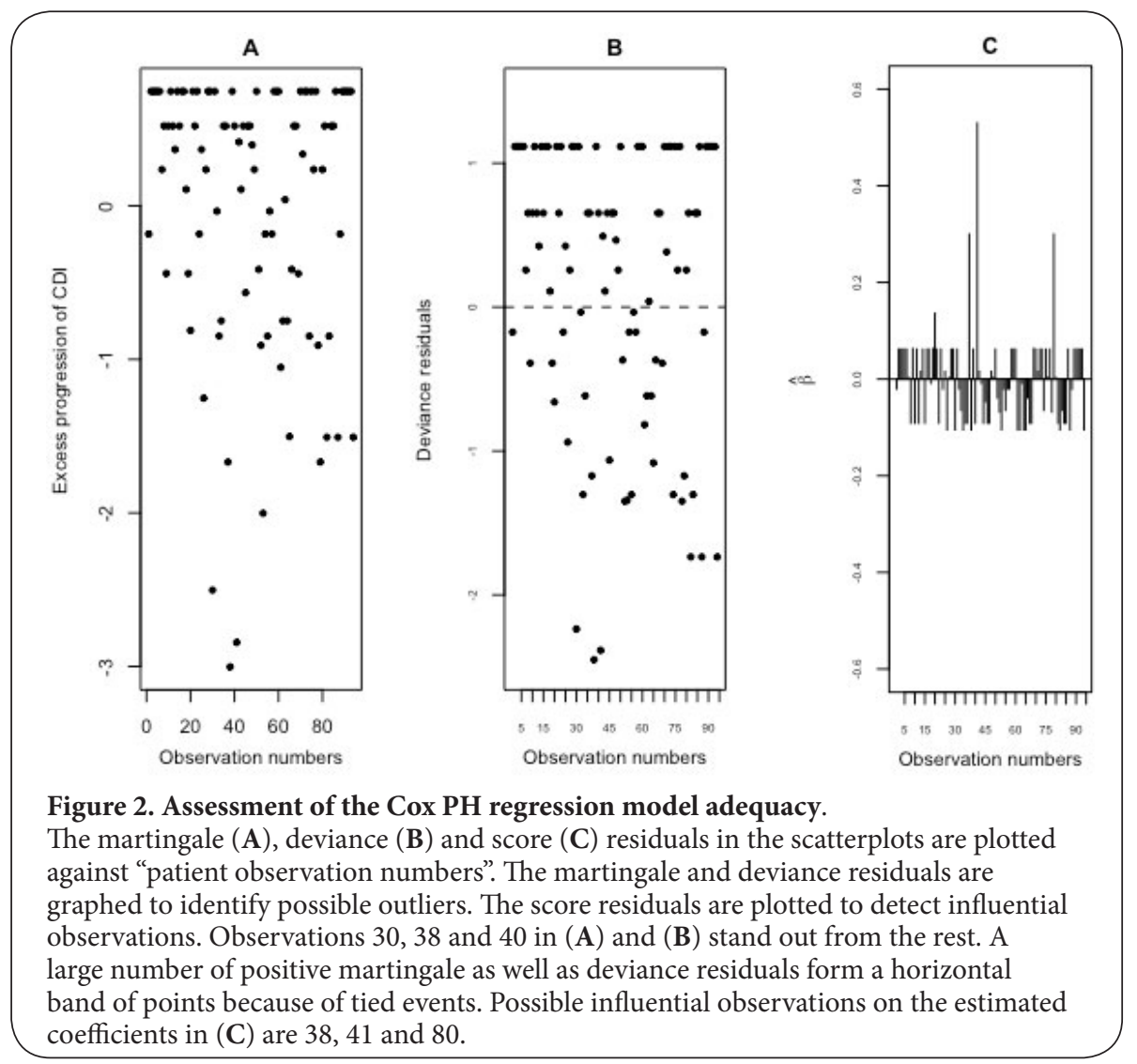

ports the currently available evidence that infusion of intestinal microorganisms into infected individuals using a retention enema is likely to be an effective therapy for the management of recurrent and/or refractory CDI. No clear superiority of a particular FMT method has yet been demonstrated, and ours is not a comparative study, thus, we do not compare the efficacy of the method used with other approaches for FMT treatments. Nevertheless, researchers claim that retention enema is an inexpensive, generally safe, effective, none invasive and simple therapy for the management of CDI [13-15].

Age seems to be an important factor affecting recurrence, resolution and number of treatments in CDI patients. We classified patients using the age threshold of 65 . This grouping was used based on a validated clinical prediction rule for recurrent $C D$ I where the age of 65 and older was established as an independent risk factor for recurrence [16]. Our results show that the proportion of patients with recurrent and/or refractory $C D I$ in the age groups was significantly different. Additionally, our results revealed that a higher proportion of younger patients were clinically resolved after treatment compared to older patients, and that older patients required more FMTs than younger patients.

The survival analysis performed on the data indicates that gender and hospitalization factors were not significantly associated with cure rates of recurrent and/or refractory $C D I$.
Conversely, survival analysis of the data substantiated age as a contributing risk factor to recurrent and/or refractory CDI resolution. We found that the hazard ratio of FMT to recurrent and/or refractory C. difficile was significantly different in clinical resolution between older and younger participants. Our finding contradicts studies that have reported that clinical resolution of CDI was not significantly different between younger and older subjects [13]. This difference is likely due to different methodologies used for data analyses. When we apply descriptive statistics to our data, we did not find significant differences in recurrent and/or refractory $C D I$ resolution between younger and older patients. However, by using survival analysis techniques, we found that the hazard of FMTs to recurrent and/or refractory $C$. difficile in younger patients was twice as high as in older patients, which suggests that older patients require more time and treatments to be clinically resolved. To our knowledge, our study is the first to apply a survival model including explanatory variables such as age group, gender and hospitalization status.

The proposed and developed models in this article seem to be adequate to analyze these type of data as supported by the graphical examinations of the residuals' normality. We identify influential subjects and provide evidence that the $\mathrm{PH}$ assumptions are satisfied. To the best of our knowledge, this is the first study to demonstrate the appropriateness of the 
proposed model for investigating CDI by hypothesis testing and detailed examination of the model's residuals.

This study has some limitations. For example, the CDI patients' data were not recorded prospectively. The analyses were based on relatively few available variables and that limits our statistical ability to evaluate multiple risk factors reported in the literature on clinical resolution of CDI. Thus, future randomized control trials with more variables are needed to confirm our findings and more deeply investigate the impact of other risk factors on resolution of recurrent and/or refractory CDI.

\section{Conclusion}

We demonstrated with a large retrospective study of patients' medical records that the delivery of FMT via a retention enema is an effective alternative therapy for recurrent and/or refractory CDI. Our results suggest that age is strongly associated with clinical resolution of recurrent and/or refractory CDI, with older patients requiring more FMTs and more time to be clinically resolved. We also showed that the Kaplan-Meier estimator and Cox proportional hazard model used to analyze data of CDI patients are adequate.

\section{Competing interests}

The authors declare that they have no competing interests.

Authors' contributions

\begin{tabular}{|l|c|c|c|}
\hline Authors' contributions & TP & PTK & AFD \\
\hline Research concept and design & -- & $\checkmark$ & -- \\
\hline Collection and/or assembly of data & $\checkmark$ & -- & -- \\
\hline Data analysis and interpretation & $\checkmark$ & -- & -- \\
\hline Writing the article & $\checkmark$ & -- & -- \\
\hline Critical revision of the article & $\checkmark$ & $\checkmark$ & $\checkmark$ \\
\hline Final approval of article & $\checkmark$ & $\checkmark$ & $\checkmark$ \\
\hline Statistical analysis & $\checkmark$ & -- & -- \\
\hline
\end{tabular}

\section{Acknowledgements and funding}

We acknowledge participant patients and the staff at the St. Joseph's Healthcare Hamilton for their cooperation. This research was partly supported by Natural Sciences and Engineering Research Council (Canada) Discovery Grants.

Publication history

Editor: Qiang Shawn Cheng, Southern Illinois University, USA. Received: 17-Nov-2019 Final Revised: 30-Dec-2019

Accepted: 16-Jan-2020 Published: 26-Jan-2020

\section{References}

1. Gorbach SL. Antibiotics and Clostridium difficile. N Eng/ J Med. 1999; 341:1690-1. | Article | PubMed

2. Kelly $\mathrm{CP}$ and LaMont JT. Clostridium difficile--more difficult than ever. $N$ Engl J Med. 2008; 359:1932-40. | Article | PubMed

3. Cohen SH, Gerding DN, Johnson S, Kelly CP, Loo VG, McDonald LC, Pepin J and Wilcox MH. Clinical practice guidelines for Clostridium difficile infection in adults: 2010 update by the society for healthcare epidemiology of America (SHEA) and the infectious diseases society of America (IDSA). Infect Control Hosp Epidemiol. 2010; 31:431-55. | Article I PubMed

4. Lee $\mathrm{CH}$, Belanger JE, Kassam Z, Smieja M, Higgins D, Broukhanski G and
Kim PT. The outcome and long-term follow-up of 94 patients with recurrent and refractory Clostridium difficile infection using single to multiple fecal microbiota transplantation via retention enema. Eur J Clin Microbiol Infect Dis. 2014; 33:1425-8. | Article | PubMed

5. Harrington DP and Fleming TR. A class of rank test procedures for censored survival data. Biometrika. 1982; 69:553-66. | Article

6. Cox DR. Regression models and life-tables. J R Stat Soc Ser B. 1972; 34:187-202. | Article

7. R Development Core Team R. A language and environment for statistical computing. 2013. | Article

8. Musher DM, Aslam S, Logan N, Nallacheru S, Bhaila I, Borchert F and Hamill RJ. Relatively poor outcome after treatment of Clostridium difficile colitis with metronidazole. Clin Infect Dis. 2005; 40:1586-90. | Article I PubMed

9. Pepin J, Saheb N, Coulombe MA, Alary ME, Corriveau MP, Authier S, Leblanc M, Rivard G, Bettez M, Primeau V, Nguyen M, Jacob CE and Lanthier L. Emergence of fluoroquinolones as the predominant risk factor for Clostridium difficile-associated diarrhea: a cohort study during an epidemic in Quebec. Clin Infect Dis. 2005; 41:1254-60. | Article I PubMed

10. Louie TJ, Miller MA, Mullane KM, Weiss K, Lentnek A, Golan Y, Gorbach $S$, Sears $P$ and Shue YK. Fidaxomicin versus vancomycin for Clostridium difficile infection. N Engl J Med. 2011; 364:422-31. | Article | PubMed

11. Gough $E$, Shaikh $H$ and Manges AR. Systematic review of intestinal microbiota transplantation (fecal bacteriotherapy) for recurrent Clostridium difficile infection. Clin Infect Dis. 2011; 53:994-1002. | Article I PubMed

12. Drekonja D, Reich J, Gezahegn S, Greer N, Shaukat A, MacDonald R, Rutks I and Wilt TJ. Fecal Microbiota Transplantation for Clostridium difficile Infection: A Systematic Review. Ann Intern Med. 2015; 162:630-8. | Article I PubMed

13. Burke KE and Lamont JT. Fecal transplantation for recurrent Clostridium difficile infection in older adults: a review. J Am Geriatr Soc. 2013; 61:1394-8. | Article | PubMed

14. Emanuelsson F, Claesson BE, Ljungstrom L, Tvede $M$ and Ung KA. Faecal microbiota transplantation and bacteriotherapy for recurrent Clostridium difficile infection: a retrospective evaluation of 31 patients. Scand J Infect Dis. 2014; 46:89-97. | Article | PubMed

15. Wang JW, Kuo CH, Kuo FC, Wang YK, Hsu WH, Yu FJ, Hu HM, Hsu PI, Wang $\mathrm{JY}$ and WU DC. Fecal microbiota transplantation: Review and update. $J$ Formos Med Assoc. 2019; 118 Suppl 1:S23-S31. | Article | PubMed

16. Hu MY, Katchar K, Kyne L, Maroo S, Tummala S, Dreisbach V, Xu H, Leffler DA and Kelly CP. Prospective derivation and validation of a clinical prediction rule for recurrent Clostridium difficile infection. Gastroenterology. 2009; 136:1206-14. | Article | PubMed

\section{Citation:}

Petukhova T, Kim PT and Desmond AF. Survival analysis to assess of the effectiveness of fecal microbiota transplantation for recurrent and/or refractory Clostridioides difficile infection. J Med Stat Inform. 2020; 8:1.

http://dx.doi.org/10.7243/2053-7662-8-1 